\title{
Meniscal Allograft Transplantation Does Not Prevent or Delay Progression of Knee Osteoarthritis
}

\author{
Catherine Van Der Straeten ${ }^{1}$, Paul Byttebier $^{2}$, Annelies Eeckhoudt ${ }^{2}$, Jan Victor ${ }^{2}$ \\ 1 Musculoskeletal Sciences and Technology, Department of Surgery and Cancer, Imperial College London, \\ London, United Kingdom, 2 Department Orthopaedics and Traumatology, Ghent University Hospital, Ghent, \\ Belgium \\ * c.van-der-straeten@imperial.ac.uk
}

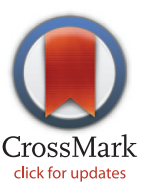

\section{G OPENACCESS}

Citation: Van Der Straeten C, Byttebier P, Eeckhoudt A, Victor J (2016) Meniscal Allograft Transplantation Does Not Prevent or Delay Progression of Knee Osteoarthritis. PLoS ONE 11(5): e0156183. doi:10.1371/journal.pone.0156183

Editor: Chunfeng Zhao, Mayo Clinic Minnesota, UNITED STATES

Received: February 17, 2016

Accepted: May 10, 2016

Published: May 26, 2016

Copyright: @ 2016 Van Der Straeten et al. This is an open access article distributed under the terms of the Creative Commons Attribution License, which permits unrestricted use, distribution, and reproduction in any medium, provided the original author and source are credited.

Data Availability Statement: Due to the presence of identifying information, anonymized data are available upon request to the Corresponding Author.

Funding: The authors have no support or funding to report.

Competing Interests: The authors have declared that no competing interests exist.
Abstract

\section{Background}

Meniscal tears are common knee injuries. Meniscal allograft transplantation (MAT) has been advocated to alleviate symptoms and delay osteoarthritis (OA) after meniscectomy. We investigated (1) the long-term outcome of MAT as a treatment of symptomatic meniscectomy, (2) most important factors affecting survivorship and (3) OA progression.

\section{Methods}

From 1989 till 2013, 329 MAT were performed in 313 patients. Clinical and radiographic results and MAT survival were evaluated retrospectively. Failure was defined as conversion to knee arthroplasty (KA) or total removal of the MAT.

\section{Results}

Mean age at surgery was 33 years (15-57); $60 \%$ were males. No-to-mild cartilage damage was found in 156 cases, moderate-to-severe damage in 130. Simultaneous procedures in 118 patients included cartilage procedures, osteotomy or ACL-reconstruction. At a mean follow-up of 6.8 years (0.2-24.3years), 5 patients were deceased and 48 lost (14.6\%), 186 MAT were in situ (56.5\%) whilst $90(27.4 \%)$ had been removed, including 63 converted to a KA (19.2\%). Cumulative allograft survivorship was $15.1 \%(95 \% \mathrm{Cl}: 13.9-16.3)$ at 24.0 years. In patients $<35$ years at surgery, survival was significantly better $(24.1 \%)$ compared to $\geq 35$ years $(8.0 \%)(p=0.017)$. In knees with no-to-mild cartilage damage more allografts survived $(43.0 \%)$ compared to moderate-to-severe damage $(6.6 \%)(p=0.003)$. Simultaneous osteotomy significantly deteriorated survival ( $0 \%$ at 24.0 years) $(p=0.010) .61 \%$ of patients underwent at least one additional surgery (1-11) for clinical symptoms after MAT. Consecutive radiographs showed significant OA progression at a mean of 3.8 years $(p<0.0001)$. Incremental Kellgren-Lawrence grade was $+1,1$ grade per 1000 days $(2,7 y r s)$. 


\section{Conclusions}

MAT did not delay or prevent tibiofemoral OA progression. 19.2\% were converted to a knee prosthesis at a mean of 10.3 years. Patients younger than 35 with no-to-mild cartilage damage may benefit from MAT for relief of symptoms (survivorship $51.9 \%$ at 20.2 years), but patients and healthcare payers and providers should be aware of the high number of surgical re-interventions.

\section{Introduction}

Meniscal tears are common injuries. The overall incidence is unknown but the surgical incidence is reported to be 60 to 70 per 100.000 per year [1-3]. Most meniscal tears are caused by the combination of tissue degeneration and high mechanical loads. Meniscal function may also be lost because of traumatic events, with or without ligament injury, or congenital malformation. Meniscectomy through arthrotomy of the knee used to be a common orthopaedic treatment for symptomatic meniscal tears [4]. The advent of arthroscopy significantly reduced the morbidity of the operative procedure [5,6]. However, the appraised virtues of this new technology including its technical elegance, decreased postoperative pain, improved aesthetics and faster recovery, lowered the threshold to enter the knee joint and perform diagnostic and therapeutic interventions, such as 'partial' meniscectomy. Consequently, the number of procedures increased exponentially [7] and nowadays patients often undergo repetitive knee arthroscopies with progressive loss of meniscal volume. In the US, the 2006 National Survey of Ambulatory Surgery reported approximately one million knee arthroscopies yearly including nearly 500,000 procedures for meniscal tears mostly partial meniscectomies [8].

After meniscectomy, the tibiofemoral contact area is decreased leading to higher contact stresses between the curved femoral condyle and the flat tibial plateau often associated with symptoms including pain and instability and a faster progression of tibiofemoral osteoarthritis (OA) [9]. The first report on the relation between meniscectomy and cartilage degeneration with bone remodeling dates back to 1948 [10]. In later years, the meniscus was recognized as an essential structure for biomechanical and biological homeostasis of the knee $[11,12]$. In a loaded, ex-vivo setting, the meniscus reduces the stress on the cartilage by load sharing of up to $50 \%$ on the medial and $70 \%$ on the lateral side [13]. The medial meniscus is an important contributor to knee stability [14] whilst both menisci play a role in shock absorption [15] and joint lubrication [16]. Recent research revealed an essential biological role of the meniscus in the development and progression of OA. In vitro pro-inflammatory stimulation of injured meniscal tissue causes production of cytokines, chemokines, matrix degrading enzymes and other catabolic factors by meniscal cells [17-19]. This effect may be enhanced by obesity and/or agerelated dysregulation of anabolic gene expression and changes in cytokine release [17,20,21]. In vivo, Petty et al demonstrated radiographic signs of OA at 8 to 16 years post-meniscectomy [22] whilst Cohen et al showed cartilage loss on MRI, 7 years after meniscectomy [23].

Meniscal substitution by meniscal allograft transplantation (MAT) has been advocated to alleviate clinical symptoms and delay the development and progression of OA especially in young people [24-26]. MAT follow-up studies have shown encouraging short-term results regarding healing of the allograft to the joint capsule [24] and symptomatic relief [25] and suggested protection of the articular cartilage [26]. However, none of them could demonstrate that MAT re-established the load distribution function of the native meniscus and was able to defer degenerative OA $[27,28]$. 
At Ghent University Hospital, MAT has been performed since 1989 as a surgical option post-meniscectomy. We conducted a retrospective study evaluating the long-term clinical results, allograft survivorship and radiographic OA progression. Additional knee interventions and determinants for success/failure were assessed. Long-term effect of meniscus substitution on the development and progression of degenerative OA was investigated. Hence the research questions were: (1) What is the long-term outcome of MAT as a treatment of symptomatic meniscectomy, (2) which are the most important factors affecting survivorship and (3) does meniscal substitution delay the development and progression of OA and the need for prosthetic reconstructive surgery?

\section{Patients and Methods}

Between 1989 and 2013, 329 MAT were performed at Ghent University Hospital by four supervising orthopaedic surgeons. Indications were young to middle-aged patients ( $<60$ years) with moderate to severe knee symptoms (pain, swelling, instability) shortly after total meniscectomy or after a failed meniscus replacement with an artificial polyurethane meniscus (8 cases) or a collagen meniscal implant (3 cases). MAT was usually performed during or shortly after complete debridement of the meniscus (total meniscectomy) in patients with recurrent symptoms after one or several partial meniscectomies. Intact donor meniscal allografts were provided by the University Hospital Tissue Bank and were matched for size, right/left knee and lateral/ medial meniscus. Donor menisci were used as fresh and viable within two weeks of procurement and storage at $37^{\circ} \mathrm{C}$ or had been freshly frozen at $-80^{\circ} \mathrm{C}$. None of the allografts were irradiated. MAT were initially performed through a mini-open surgical approach [29] but since 2007 also arthroscopically [30].

We conducted a retrospective review of all MAT. Patients consulting at the department of orthopaedics and traumatology are asked to sign a generic informed consent giving permission to use their medical data for retrospective research purposes (approved by Ghent University Hospital Ethics Committee-B670201317873).

All available demographic, surgical, clinical and radiographic data were collected retrospectively from the University Hospital Electronic Patient Dossiers (EPD). Electronic patient data recording was started in 1997. Older data from paper records were scanned and stored in the EPD and could easily be consulted. Demographic data included gender, age at surgery, weight, length, BMI and smoking history. Data related to MAT included allograft preservation method, surgical approach, lateral/medial MAT, intraoperative cartilage assessment using the Modified Outerbridge scale [31] and recording of simultaneous surgeries such as cartilage procedures (microfractures or osteochondral autograft transfer system (OATS)), high tibial osteotomy (HTO) or anterior cruciate ligament (ACL) reconstruction. Clinical data included postoperative complications, knee symptoms and subsequent treatments. Additional operations at the index knee performed at the University Hospital were registered. Only the surgical interventions related to knee morbidity were analyzed. Second-look arthroscopies for suture removal or allograft biopsies for DNA testing [32] were not taken into account, because we wanted to focus on re-interventions for clinical symptoms related to the MAT. All available pre-transplantation and consecutive postoperative radiographs were rated according to the KellgrenLawrence radiographic $\mathrm{OA}$ scale [33] by two investigators ( $\mathrm{PB}, \mathrm{AE})$ independently and their evaluations compared for consistency with the radiologist's report at the time. Patients who had not been seen at the clinic recently were contacted by phone, and were asked whether they agreed to answer a few questions on their MAT. This additional oral consent was noted in the study spreadsheet. Questions included whether the allograft was still in situ, removed and/or replaced, and if they had undergone additional knee surgeries at other hospitals. Patients with 
MAT in situ were asked to rate their current pain on a VAS scale from 0 to $10(0=$ no pain; $10=$ extreme pain) and to describe any other knee symptoms. Besides, patient satisfaction was recorded on a VAS scale from 0 to $10(0=$ not satisfied at all; $10=$ very satisfied $)$. Patients were considered 'lost to follow-up' if they were not traceable even after very intensive search. Failure of MAT was defined as total removal of the allograft either by total allograft meniscectomy or during conversion to a total knee arthroplasty (TKA) or a unicompartmental knee arthroplasty (UKA) [34]. Partial allograft meniscectomy, tears (sutured or not), degeneration of the donor meniscus visible on MRI or arthroscopically, meniscal extrusion, and symptomatic knees needing additional treatment were not considered a failure as long as the allograft was not totally removed.

Statistical analyses were performed using the IBM-SPSS Statistics 22 software (SPSS, an IBM Company, Chicago, IL, USA) and SAS/STAT Statistical Analysis Software (SAS Institute Inc., Cary, NC, USA). Statistical tests were verified by a professional statistician (GB). Level of statistical significance used was 0.05 .

The research questions were examined as follows: (1) Life tables and Kaplan-Meier survivorship analysis was performed for the total cohort. For the allografts in situ, outcome scores including pain and satisfaction rating by the patients, meniscal re-interventions and other subsequent knee surgeries were analysed with descriptive statistics. Comparison between different outcome groups, age groups, cartilage damage and radiographic OA grading groups regarding allograft time in situ was performed using parametric and non-parametric statistics as appropriate. (2) In order to establish determinant patient and procedure factors sub-analysis was performed by gender, age at surgery, knee compartment cartilage damage, patient BMI and smoking history, allograft preservation method, open versus arthroscopic surgery, lateral versus medial meniscal transplantation and concomitant operative procedures. Comparison of survival curves of subgroups was performed using log rank (Mantel-Cox) test. Cox proportional-hazards regression was used to establish most important determining factors for survival. (3) The evolution of OA progression was evaluated by comparing Kellgren-Lawrence OA grade on consecutive X-rays in knees with MAT in situ. The difference of radiographic OA grading between consecutive X-rays was assessed with non-parametric statistics and plotted using a linear regression model (Influence Statistics) [35]. Difference in grading was also plotted against time and estimated time to increase at least one Kellgren-Lawrence grade was computed.

\section{Results}

329 MAT were implanted in 313 patients from 1989 till 2013. In 6 cases the MAT was replaced by a new allograft after failure, 5 patients received a MAT in both knees and 5 patients a lateral and medial MAT at the same knee (Table 1). Mean age at surgery was 33.3 years (15-57 years). Sixty percent of patients were male. Cartilage damage was assessed during the operation as no to mild cartilage damage (Outerbridge grade $<$ III) in 156 cases $(47.4 \%$ ) and moderate to severe damage (grade $\geq \mathrm{III}$ ) in 130 cases (39.5\%); not noted intraoperatively in 43 cases (13.1\%). There was a significant correlation between age and cartilage damage $(r=0.280 ; p<0.0001)$. Simultaneous concomitant surgeries in 118 cases (35.8\%) included cartilage procedures in 52 knees (15.8\%) (50 microfractures; 2 OATS), HTO in 39 cases (11.9\%) and ACL reconstruction in 27 knees (8.2\%). Postoperative complications occurred in 18 cases (5.5\%): 6 septic arthritis, 1 severe synovitis, possibly associated with allograft rejection, 1 deep venous thrombosis, 2 cases of algoneurodystrophy, 3 arthrofibrosis needing a mobilisation under general anaesthesia and 4 complications related to HTO (3 non-unions and 1 osteomyelitis).

(1) At the time of the retrospective review (locked-down on April $1^{\text {st }} 2015$ ), the mean follow-up was 6.8 years (median 5.2 years-range: 2 months to 24.3 years). The outcome of MAT 
Table 1. Patient Demographics: 329 Meniscal Allograft Transplantations in 313 patients.

\begin{tabular}{|c|c|c|}
\hline Gender & Male: $187(60 \%)$ & Female: $126(40 \%)$ \\
\hline Age at surgery & \multicolumn{2}{|c|}{ Mean: 33,3 years ( $15-57$ years) } \\
\hline BMI & Mean: 24.9 (16.9-35.7) & $9.1 \%$ obese $(\mathrm{BMI} \geq 30.0)$ \\
\hline Knee & Right: 180 (55\%) & Left: $149(45 \%)$ \\
\hline Meniscus & Lateral: $210(64 \%)$ & Medial: $119(36 \%)$ \\
\hline Allograft preservation & Viable: 137 (41.6\%) & Freshly frozen: $168(51.1 \%)$ \\
\hline Surgical approach & Open: 258 (78\%) & Arthroscopic: 71 (22\%) \\
\hline Cartilage: modified Outerbridge grading & Grade < III: $156(47.4 \%)$ & Grade $\geq 111: 130$ (39.5\%) \\
\hline Concomitant surgeries Simultaneous: $118(35.8 \%)$ & \multicolumn{2}{|c|}{$\begin{array}{l}\text { Cartilage (microfractures or OATS): } 52 \text { (15.8\%); High Tibial Osteotomy: } 39 \\
(11.9 \%) \text {; fACL reconstruction: } 27(8.2 \%)\end{array}$} \\
\hline
\end{tabular}

doi:10.1371/journal.pone.0156183.t001

at last follow-up is summarized in Fig 1. Five patients died of unrelated causes and 48 (14.6\%) were lost to follow-up. Ninety meniscal allografts (27.4\%) had been removed or replaced after a mean of 8,5 years in situ (median 6,6 years-range 2 months to 24,0 years). Reasons for failure included pain, synovitis, knee dysfunction, meniscus tear or re-tear. There were 63 conversions to a knee prosthesis (19.2\%) for OA progression: 48 TKA and 15 UKA. At the closure of the investigation 3 of these TKA had already been revised, including one for infection and 3 UKA had been converted to a TKA. In 8 additional patients with the allograft in situ a knee prosthesis was planned in the course of 2015 (7 TKA and 1 UKA) which will increase the total number of conversions to knee arthroplasty to $71(21.6 \%)$. In 27 cases $(8.2 \%)$ a total meniscectomy was performed for clinical symptoms with or without extrusion of the graft on MRI, followed by a re-transplantation with a new MAT in 6 cases.

Time till conversion to a TKA was significantly longer (mean 11.5 years; range $0.5-24.0$ years; SD 6.55) compared to a UKA (mean 6.5 years; range $0.7-13.8$ years; SD 4.47$)(\mathrm{p}=0.002)$

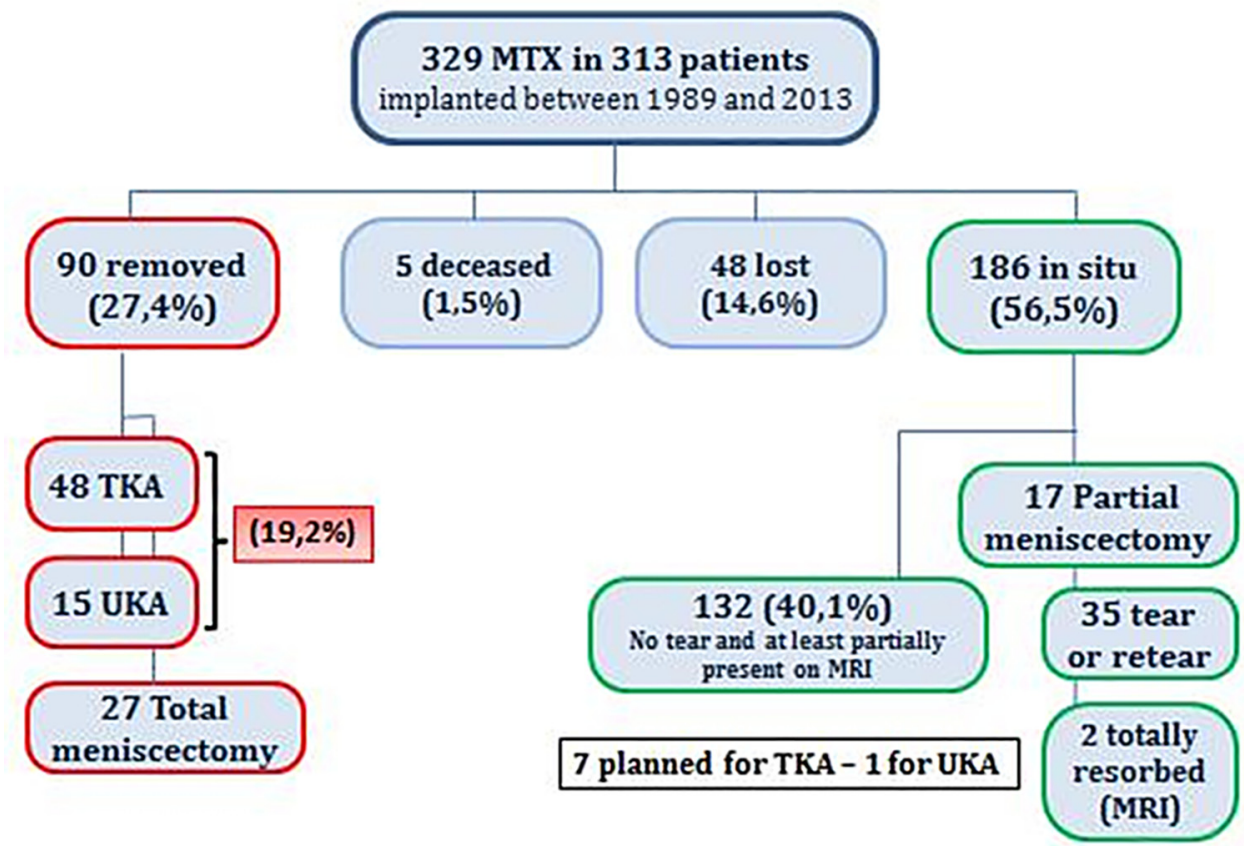

Fig 1. Outcomes of meniscal allograft transplantations (MAT). 


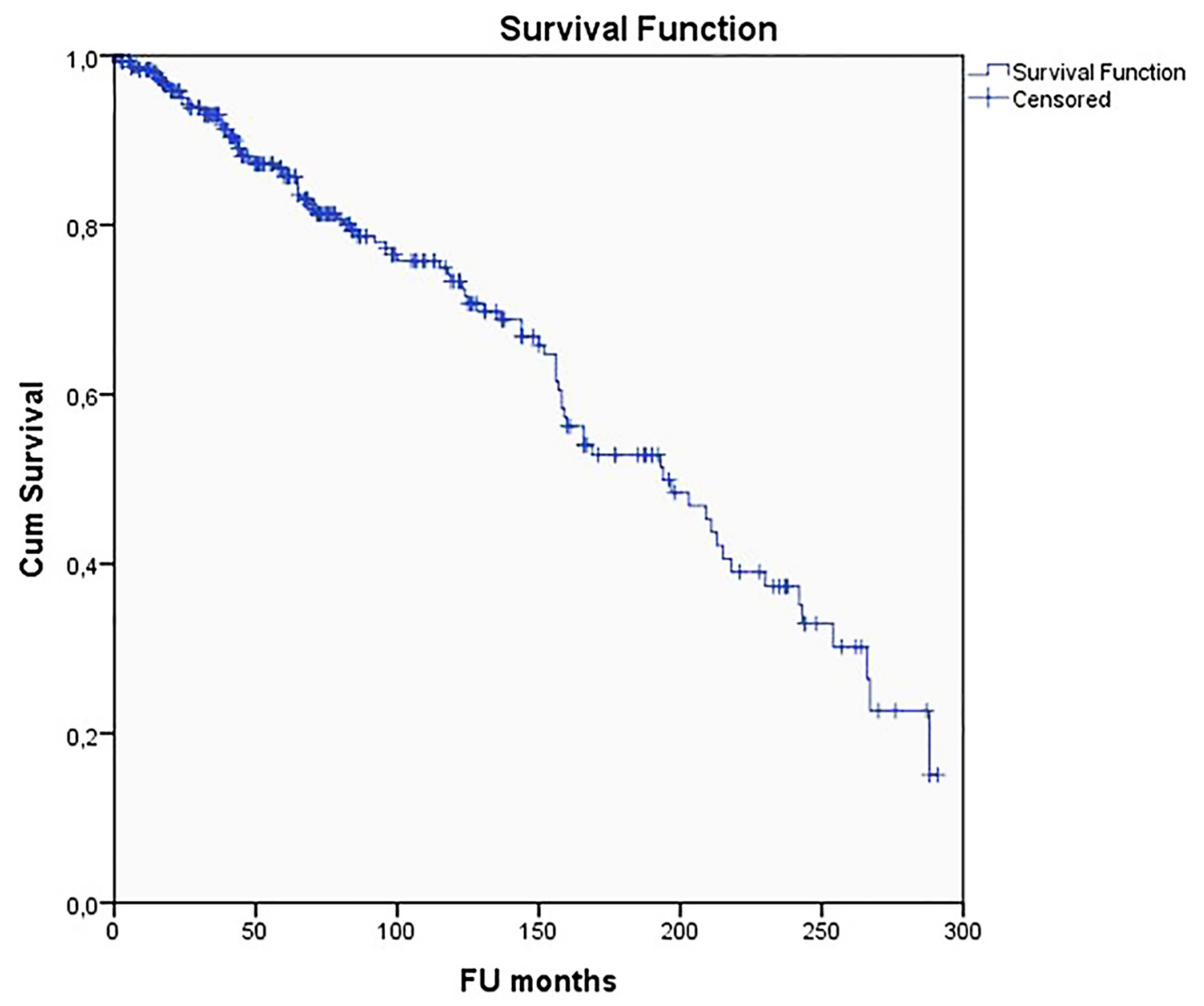

Fig 2. Kaplan-Meier cumulative survivorship of all MAT: endpoint removal of the allograft by total meniscectomy or during conversion to a knee arthroplasty (TKA or UKA).

doi:10.1371/journal.pone.0156183.g002

or a total allograft meniscectomy (mean 3.7 years; range $0.2-13.8$ years; SD 3.86) $(\mathrm{p}<0.001)$. Mean age at conversion to a TKA was 48.7 years (21-68 years; SD 9.17), and at conversion to a UKA 43.7 years (34-56 years; SD 6.39).

102 patients with an intact MAT in situ (33\%) (no tear, resorption or partial meniscectomy), reported a median VAS for pain of 5 (mean 4.3; SD 2.7) and a median VAS for satisfaction of 8 (mean 6.7; SD 3.7) at 6.6 years mean follow-up (1.0-24.0 years). Sixteen mentioned recurrent locking, popping, instability, swelling and/or stiffness of the knee.

Cumulative MAT survivorship with endpoint removal of the allograft (total meniscectomy or conversion to a knee arthroplasty) was $15.1 \%$ (95\%CI: 13.9-16.3\%) at 24.0 years (Fig 2). Mean survival time was 15.2 years.

(2) There was no significant difference in MAT survivorship between genders $(p=0.552)$, right or left knee $(\mathrm{p}=0.080)$, lateral or medial MAT $(\mathrm{p}=0.837)$, BMI categories $(\mathrm{p}=0.211)$, smokers or non-smokers $(\mathrm{p}=0.235)$ and MAT preservation method (viable or fresh-frozen) $(\mathrm{p}=0.118)$. Open versus arthroscopic surgical approach had no significant influence on survival so far ( $\mathrm{p}=0.851)$ but the longest follow-up time was only 7.4 years for the arthroscopic approach compared to 24.3 years for the open approach $(\mathrm{p}<0.001)$. In patients younger than 35 at surgery, survival was significantly better (24.1\% (95\%CI: $21.4-26.7 \%)$ ) at 24 years; mean 


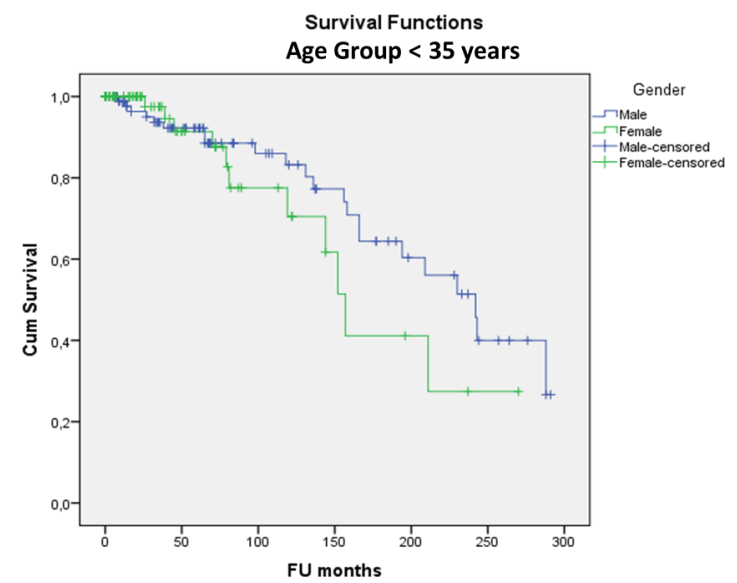

Fig 3. Kaplan-Meier cumulative survivorship of MAT in the < 35 years at surgery group-gender: no statistically significant difference between males (blue line) and females (green line) $(p=0.262$ ).

doi:10.1371/journal.pone.0156183.g003

survival time 16.7 years) compared to older patients (8.1\% (95\%CI: 7.2-9.0\%)) at 24 years; mean survival time 13.8 years) $(\mathrm{p}=0.017)$. The odds ratio of MAT failure was 2.3 in the $\geq 35$ years group compared to the $<35$ group. Yet, even in the group $<35$ years at surgery, $12 \%$ had been converted to a KA (15TKA/6UKA) at a mean of 13 years post-MAT for TKA and 9 years post-MAT for UKA. In the group $<35$ years at surgery there was no difference in survivorship between genders (at 24 years Males: 26.6\% (95\%CI: 23.3-29.9\%); Females: 27.4\% (95\%CI: $21.2-33.6 \%)-\mathrm{p}=0.262$ ) (Fig 3) or lateral versus medial meniscus (at 24 years Lateral: $37.4 \%$ (95\%CI: 31.5-43.3\%) Medial: 18.9\% (95\%CI: 15.8-22.0\%)—p = 0.738) (Fig 4).

In knees with no-to-mild cartilage damage (Outerbridge grade $<$ III) more allografts survived (43.0\% (95\%CI: 38.4-47.6\%) at 24 years; mean survival time 17.6 years) compared to moderate-to-severe damage (Outerbridge grade $\geq \mathrm{III})(6.6 \%$ (95\%CI: $5.8-7.4 \%)$ at 24 years; mean survival time 13.4 years) ( $\mathrm{p}=0.003$ ). The odds ratio of MAT failure was 3.7 in the grade $\geq$ III group compared to $<$ III. In the group with no to mild cartilage damage at surgery there was no difference in survivorship between genders (Males: 41.3\% (95\%CI: 36.0-46.6\%) Females: $59.4 \%(95 \% \mathrm{CI}: 48.5-70.3 \%)$ at 24 years $-\mathrm{p}=0.901)($ Fig 5$)$ or lateral versus medial

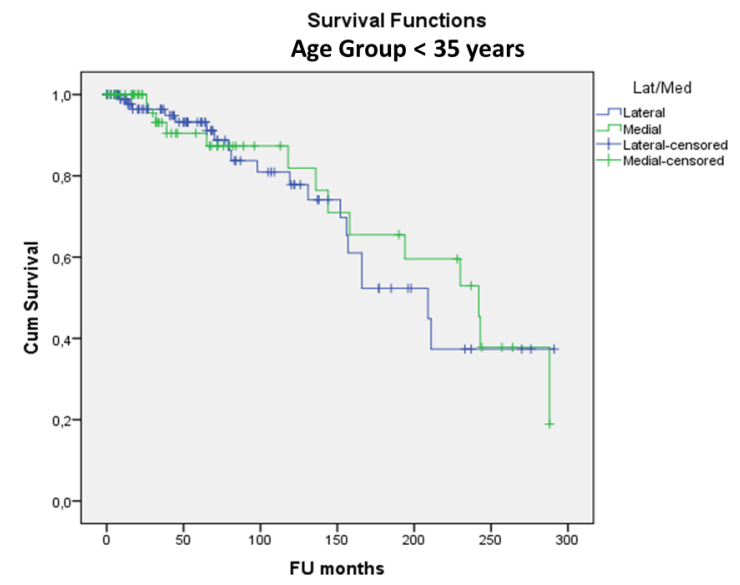

Fig 4. Kaplan-Meier cumulative survivorship of MAT in the $<35$ years at surgery group-lateral/medial: no statistically significant difference between lateral (blue line) and medial (green line) MAT $(p=0.738)$.

doi:10.1371/journal.pone.0156183.g004 


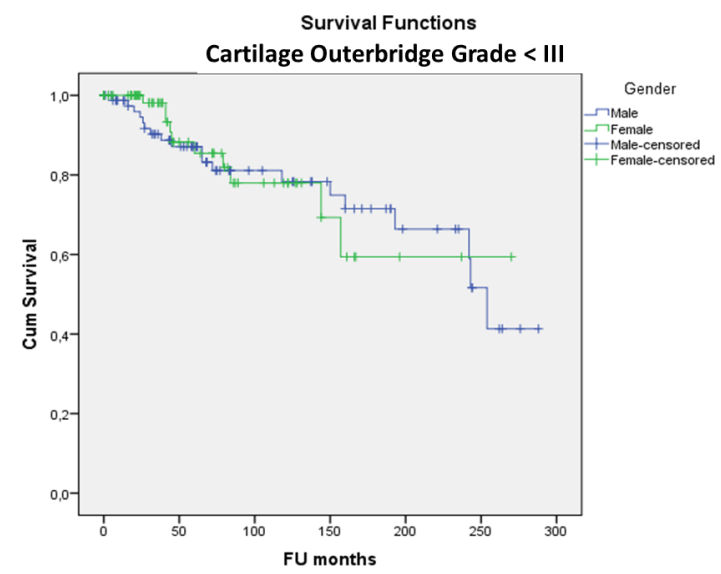

Fig 5. Kaplan-Meier cumulative survivorship of MAT in the cartilage Outerbridge grade < III groupgender: no statistically significant difference between males (blue line) and females (green line) $(p=0.901)$.

doi:10.1371/journal.pone.0156183.g005

meniscus (Lateral: 69.8\% (95\%CI: 60.7-78.9\%) Medial: 31.5\% (95\%CI: 26.7-36.3\%) at 24 years $-\mathrm{p}=0.591)($ Fig 6$)$.

Simultaneous HTO significantly deteriorated survival ( $0 \%$ at 24.0 years, mean survival time 11.2 years, range: 2 months to 24 years $)(p=0.010)$. Concomitant microfractures or ACL reconstruction did not have a significant influence on MAT survivorship ( $p=0.983$ and $\mathrm{p}=0.272$ respectively). In the group with concomitant microfractures 11 out of 50 were converted to a TKA $(22 \%)$.

$61 \%$ of patients underwent one or more surgical re-interventions ( $30 \%$ two or more, $14 \%$ three or more subsequent operations; mean 2.0; range 1 to 11) at the University Hospital for clinical symptoms at the index knee after MAT. Some patients underwent multiple further knee surgeries at other hospitals. Cox regression analysis indicated that age $\geq 35$ years at surgery and cartilage grade $\geq$ III were the most important determinants of failure of MAT. Best case scenario were patients $<35$ years at surgery with no to little cartilage damage (Outerbridge grade $<$ III) $(\mathrm{n}=85)$ with a cumulative survivorship of $51.9 \%(95 \%$ CI $45.0-58.8 \%)$ at 20.2 years

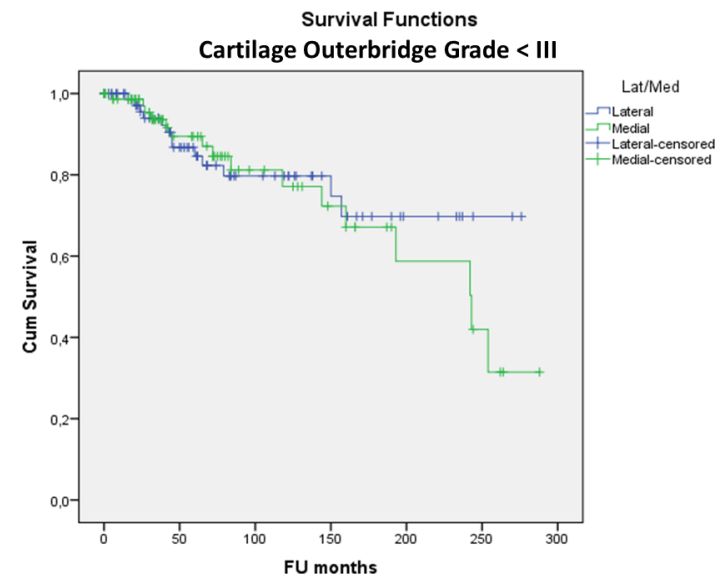

Fig 6. Kaplan-Meier cumulative survivorship of MAT in the cartilage Outerbridge grade < III grouplateral/medial: no statistically significant difference between lateral (blue line) and medial (green line) $\operatorname{MAT}(p=0.591)$.

doi:10.1371/journal.pone.0156183.g006 


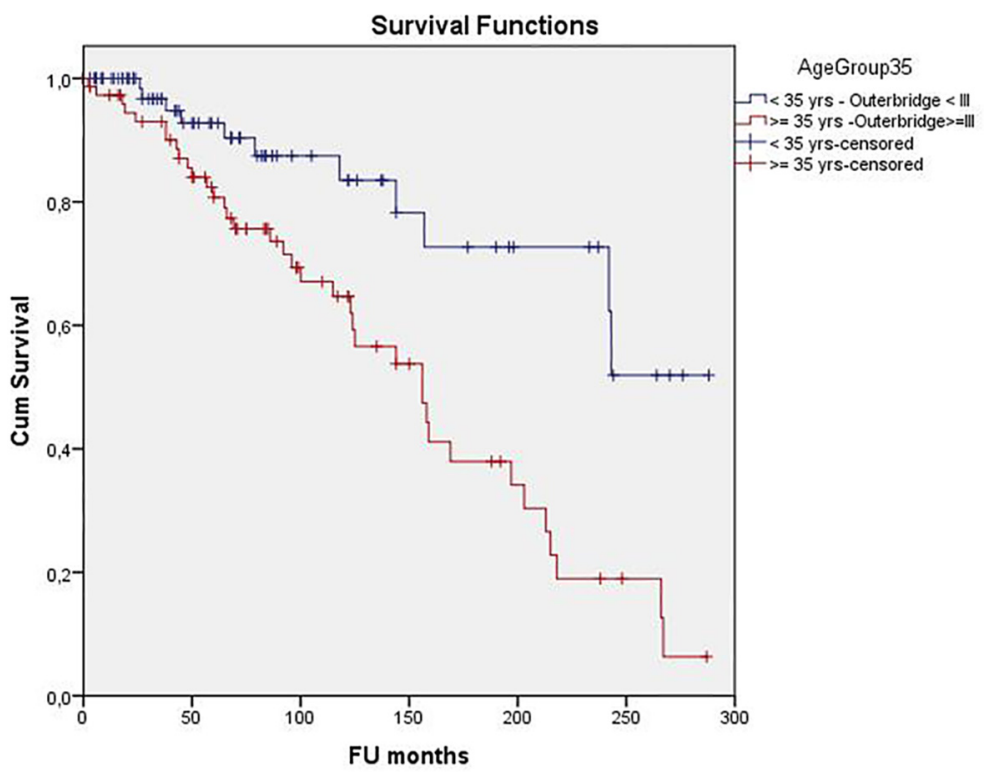

Fig 7. Kaplan-Meier survivorship curve (blue line) of the best case scenario (age $<35$ years at surgery and cartilage Outerbridge grade < III: $n=85$ ) versus Kaplan-Meier survivorship curve (red line) of the worst case scenario (Age $\geq 35$ years at surgery and Cartilage Outerbridge grade $\geq \mathrm{III}: \mathbf{n = 7 7}$ ).

doi:10.1371/journal.pone.0156183.g007

(mean survival 19 years) whilst worst case scenario were patients $\geq 35$ at surgery with moderate to severe cartilage damage (grade III-V) $(\mathrm{n}=77)$ with a survival of $6.3 \%(95 \% \mathrm{CI} 5.3-7.3 \%)$ at 22.3 years (mean survival time 12.7 years) (Fig 7 ).

(3) Consecutive standard radiographs of 107 knees with MAT in situ showed significant tibiofemoral OA progression on the Kellgren-Lawrence scale with time $(\mathrm{p}<0.0001)$ (mean 1385 days (3.8 years); range 1 month to 21.6 years; SD 4.2 years - no evolution in $40.2 \%$; grade +1 in $34.6 \%$; grade +2 in $20.6 \%$; grade +3 in $4.7 \%$ ) (Fig 8 ). The mean incremental gradient was +1.1 Kellgren-Lawrence grade per 1000 days (2,7yrs) (Fig 9). In cases with concomitant microfractures, there was a trend towards a slower evolution of OA with an increased Kellgren-Lawrence grade of +0.7 per 1000 days. The group of patients who evolved more than 2 grades on the Kellgren-Lawrence scale had a higher BMI distribution $(\mathrm{p}=0.02)$. There was no significant difference in progression of OA on the Kellgren-Lawrence scale with age $(\mathrm{p}=0.109)$ (Fig 10), gender $(\mathrm{p}=0.951)$ or lateral versus medial MAT $(\mathrm{p}=0.539)$.

\section{Discussion}

Meniscus substitution with a MAT has been advocated to alleviate symptoms of pain and instability after (partial) meniscectomy and to delay OA progression [24-26]. Pengas et al prospectively followed a cohort of 53 adolescents who had undergone meniscectomy [36]. Additional surgery was not performed till OA progression necessitated arthroplasty. At a mean follow-up of 40 years, $13.2 \%$ had received a TKA. The relative risk of OA assessed radiographically with the Kellgren-Lawrence scale was 4.5 compared to the non-operated knee at 40 years [36]. Partial meniscectomy was also shown to increase the risk for development of OA although less than total meniscectomy [37]. The current retrospective study of MAT was undertaken to evaluate the outcome of meniscal replacement after meniscectomy and to assess whether progression of knee OA was effectively delayed or prevented. The main strength of our study is that it comprises the largest patient cohort treated with MAT with the longest follow-up. 


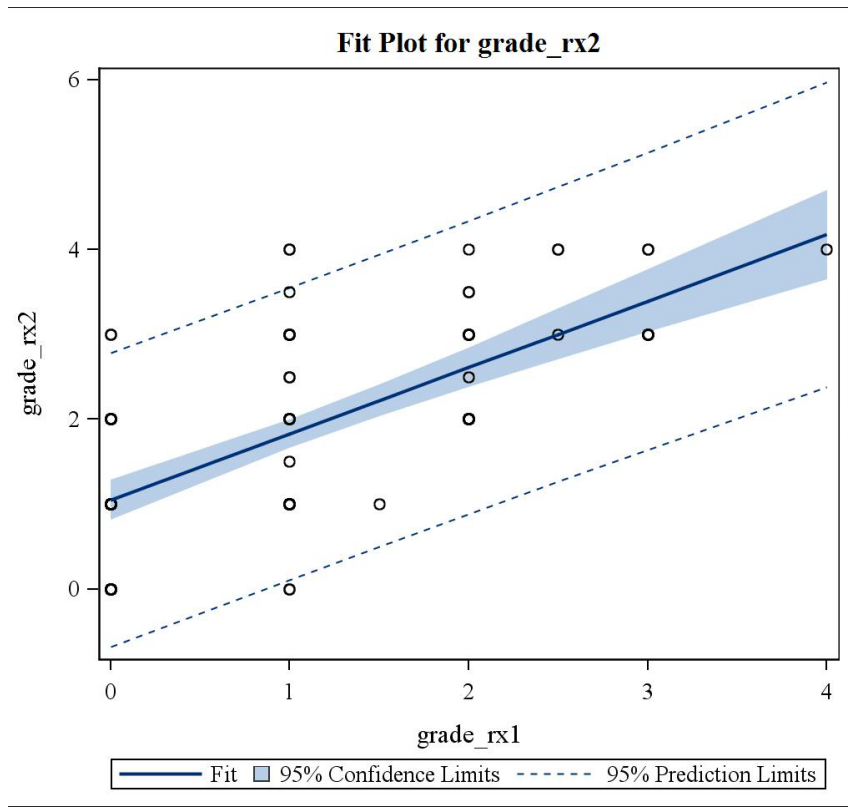

Fig 8. Fit-plot regression curve for evolution of Kellgren-Lawrence grade on consecutive radiographs.

doi:10.1371/journal.pone.0156183.g008

The study has several limitations. Due to its retrospective nature and the long-term followup up to 24 years, $14.6 \%$ of patients were lost and prospective clinical scores were not available.

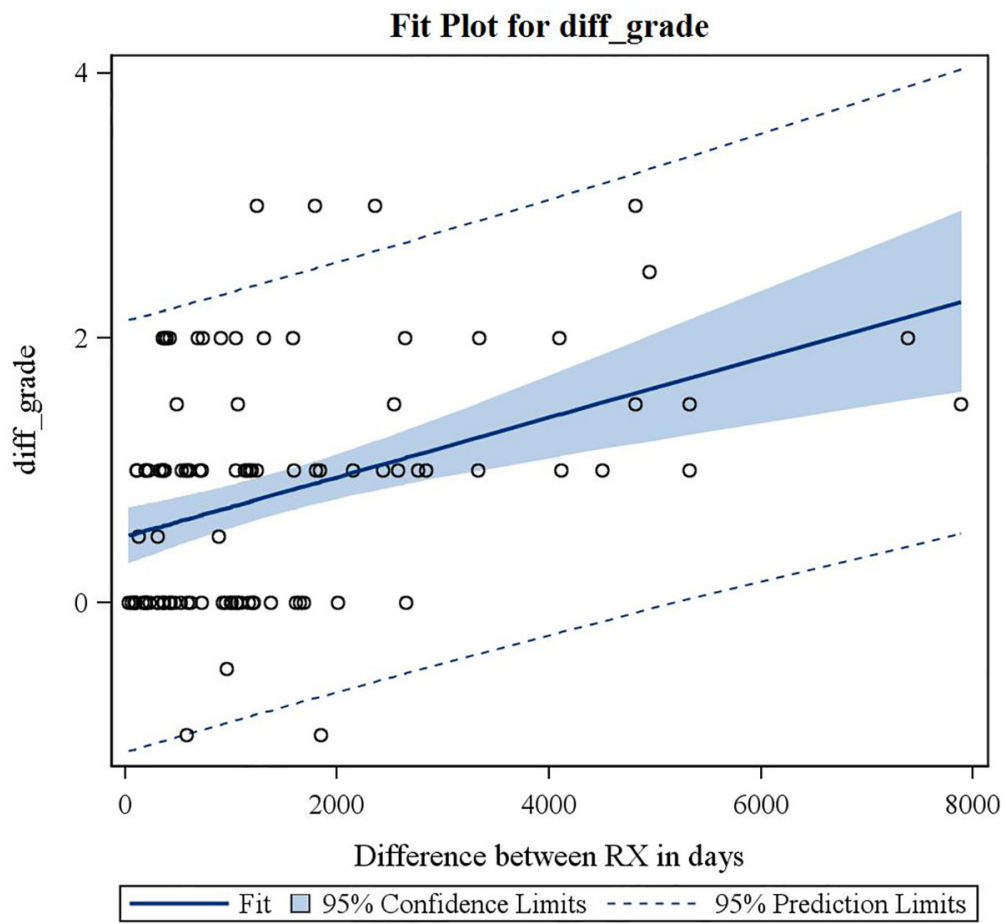

Fig 9. Fit-plot regression curve for difference in Kellgren-Lawrence grade on consecutive radiographs over time.

doi:10.1371/journal.pone.0156183.g009 


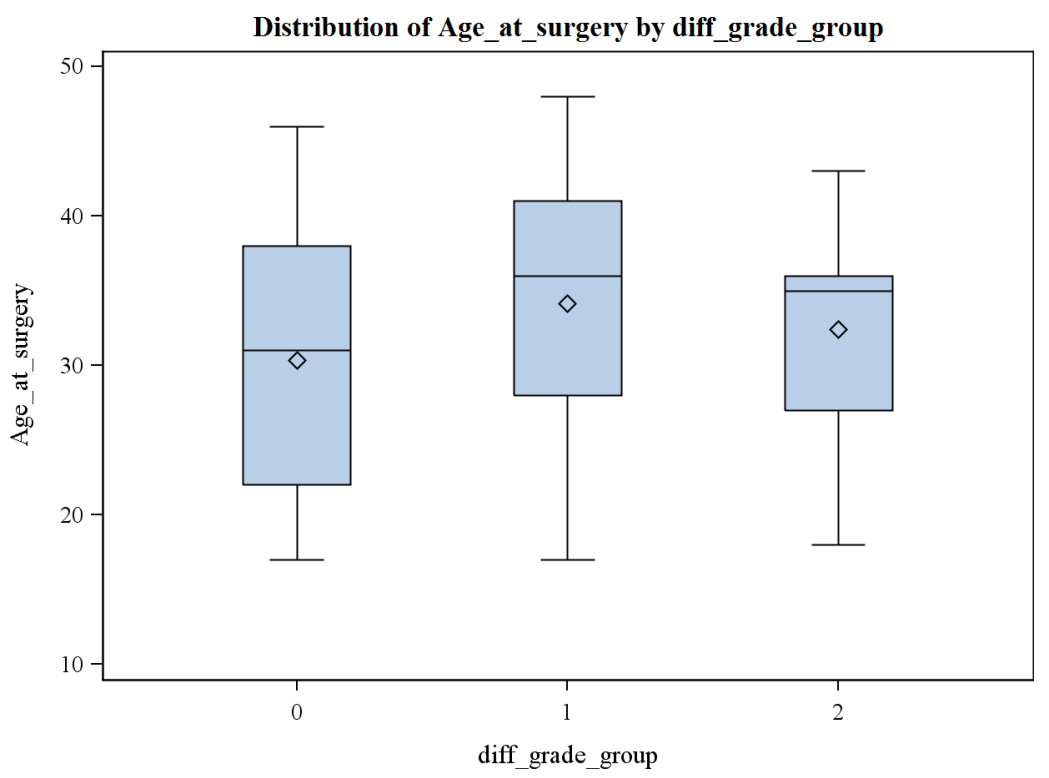

Fig 10. Distribution of age versus progression of grade of radiographic OA on the Kellgren-Lawrence scale $(+0,1$, or 2 grades): no statistically significant difference $(p=0.109)$.

doi:10.1371/journal.pone.0156183.g010

However, despite the complexity of the retrospective evaluation, it is important to establish a comprehensive record of MAT as a treatment involving human donor transplant material. A second limitation consists in the absence of a control group receiving conservative treatment [38-40]. Several authors have demonstrated comparable relief of clinical symptoms with physical therapy versus partial meniscectomy [38-40]. Concurrently, our results demonstrate that meniscal substitution constitutes only a temporary solution for relief of symptoms after meniscectomy. In our study, MAT was usually performed during or shortly after complete debridement of the meniscus (total meniscectomy) in patients with recurrent symptoms after one or several partial meniscectomies. As the initial partial meniscectomies were often performed at other hospitals, we have no accurate data on the time between the first partial meniscectomy and the MAT. A third limitation is that OA progression was evaluated only by use of the Kellgren-Lawrence scale on standard radiographs. A standardized, consecutive MRI assessment of the articular cartilage was not available for most patients. A prospective MRI study would provide an earlier and more accurate short-term evaluation of OA evolution [41]. However, Zaffagnini et al showed no significant difference in 5 years progression of cartilage damage on MRI after meniscal substitution with a Collagen Meniscal Implant (CMI) compared to meniscectomy without meniscal replacement [42]. Moreover, in our study, we were able to demonstrate $\mathrm{OA}$ advancement on consecutive standard radiographs, indicating definite cartilage deterioration. Besides, progression of OA led to $19.1 \%$ conversions to a knee arthroplasty at a mean of 11.5 years for TKA and 6.5 years for UKA.

In answer to our research questions, we found that (1) Overall cumulative survivorship of MAT was $15.1 \%$ at 24 years with a mean survival of 15.2 years. Over $50 \%$ of patients with the MAT in situ reported knee pain and/or other symptoms but most of them were satisfied with the outcome. However, the high number of subsequent knee interventions for symptoms, tear or failure of the MAT is concerning as it was associated with morbidity, (temporary) disability and socio-economic costs. In this regard, it is worth considering that Katz et al showed no difference in relief of symptoms between partial meniscectomy and physical therapy versus physical therapy alone in patients with meniscal tear and OA [40]. Besides, Sihvonen et al 
demonstrated that partial meniscectomy as a treatment of degenerative meniscal tears was not superior to sham arthroscopy [43].

(2) Most important determinants of success of the MAT were age younger than 35 years at surgery and no to mild cartilage damage (Outerbridge grade $<$ III). Concomitant HTO had a negative effect on survival. We could not prove a significant positive effect of simultaneous cartilage procedures on MAT survival although concomitant microfractures were associated with a trend to slower radiographic OA progression.

(3) Delay or prevention of OA by meniscal substitution after meniscectomy could not be demonstrated in this study. On the contrary, there was a significant radiographic evolution of OA. Overall, 63 MAT (19.2\%) were converted to a knee prosthesis whilst 8 additional knee arthroplasties were planned (total 71 or $21.6 \%$ ). Even in the group $<35$ years at surgery, 21 MAT (12\%) were converted to a knee prosthesis (15TKA/6UKA) at a mean of 13 years postMAT for TKA and 9 years for UKA. These results are comparable to the report of 13.2\% TKA at a mean of 40 years after meniscectomy in adolescents who did not receive any additional surgical treatment [36]. In our study, the mean age of patients at knee arthroplasty was substantially younger ( 48.7 years for TKA; 43.7 years for UKA) compared to large series of TKA and UKA reporting mean ages at surgery of over 60 years [44,45]. In the group less than 35 years at MAT, who were converted to a KA, the mean age at knee arthroplasty was even younger: 43.2 years (21-57) for TKA and 40.7 years (34-44) for UKA.

Our results regarding OA progression are concordant with a meta-analysis concluding that the majority of studies could not demonstrate a protective effect of MAT on cartilage and that therefore, based on the current literature, MAT does not prevent or delay OA [46]. Another literature review of 24 studies cautioned that MAT may delay the progression of damaged cartilage but does not prevent degeneration of previously healthy cartilage [47]. Whether these findings are related to cartilage degeneration initiated by meniscal injury [19] or by repetitive surgical trauma [41], or to the inability of the MAT to reproduce the biomechanical and biological function of the native meniscus, remains to be investigated.

\section{Conclusions}

Our study indicates that meniscal allograft transplantation (MAT) performs well in patients younger than 35 with no-to-mild cartilage damage. These patients may benefit from MAT for relief of symptoms, but patients and healthcare payers and providers should be aware of the high number of surgical re-interventions. There is no evidence that MAT prevents or delays tibiofemoral OA progression.

\section{Acknowledgments}

We thank Geert Byttebier for his professional statistical assistance.

The research was conducted at the Department of Orthopaedics and Traumatology, Ghent University Hospital, Ghent Belgium

\section{Author Contributions}

Conceived and designed the experiments: CVDS JV. Performed the experiments: CVDS PB AE JV. Analyzed the data: CVDS PB AE JV. Contributed reagents/materials/analysis tools: CVDS JV. Wrote the paper: CVDS JV.

\section{References}

1. Poulsen MR, Johnson DL. Meniscal injuries in the young, athletically active patient. Phys Sportsmed. 2011 Feb; 39(1):123-30 doi: 10.3810/psm.2011.02.1870 PMID: 21378495 
2. Hede A, Jensen DB, Blyme P, Sonne-Holm S. Epidemiology of meniscal lesions in the knee. 1,215 open operations in Copenhagen 1982-84. Acta Orthop Scand. 1990 Oct; 61(5):435-7. PMID: 2239168

3. Baker BE, Peckham AC, Pupparo F, Sanborn JC. Review of meniscal injury and associated sports. Am J Sports Med. 1985; Jan-Feb 13(1):1-4. PMID: 3838420

4. Smillie IS. The current pattern of internal derangements of the knee joint relative to the menisci. Clin Orthop Relat Res. 1967 Mar-Apr; 51:117-22. PMID: 6027008

5. Carr A, Price AJ, Glyn-Jones S, Rees JL. Advances in arthroscopy-indications and therapeutic applications. Nature Reviews Rheumatology 2015; 11; 77-85 doi: 10.1038/nrrheum.2014.174 PMID: 25348038

6. Katz JN, Losina E. Arthroscopic partial meniscectomy for degenerative tears: where do we stand? Osteoarthritis and Cartilage November 2014; 22 (11), 1749-1751

7. Kim S, Bosque J, Meehan JP, Jamali A, Marder R. Increase in outpatient knee arthroscopy in the United States: a comparison of National Surveys of Ambulatory Surgery, 1996 and 2006. J Bone Joint Surg (Am) 2011, 93(11):994-1000

8. Hall MJ, Golosinskij A, Cullen KA. Ambulatory surgery in the United States, 2006. Natl Health Stat Report 2009 Vol 0 (1-25)

9. Englund M, Lohmander LS. Risk Factors for Symptomatic Knee Osteoarthritis Fifteen to Twenty-Two Years after Meniscectomy. Arthr Rheum Vol. 50, No. 9, September 2004, 2811-2819.

10. Fairbank TJ. Knee joint changes after meniscectomy. J Bone Joint Surg Br. 1948 Nov; 30B(4):664-70. PMID: 18894618

11. Dye SF. The knee as a biologic transmission with an envelope of function: a theory. Clin Orthop Relat Res. 1996 Apr;(325: ):10-8. PMID: 8998861

12. Kurosawa $\mathrm{H}$, Fukubayashi $\mathrm{T}$, Nakajima $\mathrm{H}$. Load-bearing mode of the knee joint: physical behavior of the knee joint with or without menisci. Clin Orthop Relat Res. 1980 Jun;(149: ):283-90 PMID: 7408313

13. Fukubayashi $\mathrm{T}$, Kurosawa $\mathrm{H}$. The contact area and pressure distribution pattern of the knee. A study of normal and osteoarthrotic knee joints. Acta Orthop Scand. 1980 Dec; 51(6):871-9. PMID: 6894212

14. Warren RF. Arthroscopic meniscus repair. Arthroscopy. 1985; 1(3):170-2 PMID: 3841638

15. Seedholm B, Hargreaves DJ. Transmission of the Load in the Knee Joint with Special Reference to the Role of the Menisci: Part II: Experimental Results, Discussion and Conclusions. Archive Engineering in Medicine 1971-1988 (vols 1-17) 10/1979; 8(4):220-228.

16. MacConaill MA. Lubrication of Mammalian Joints J. Anat., 66, 210 (1932)

17. Stone A, Loeser RF, Vanderman KS, Long DL, Clark SC, Ferguson CM. Pro-inflammatory stimulation of meniscus cells increases production of matrix metalloproteinases and additional catabolic factors involved in osteoarthritis pathogenesis. Osteoarthritis Cartilage. 2014 February; 22(2): 264-274. doi: 10.1016/j.joca.2013.11.002 PMID: 24315792

18. McNulty AM, Moutos FT, Weinberg B, Guilak F. Enhanced Integrative Repair of the Porcine Meniscus In Vitro by Inhibition of Interleukin-1 or Tumor Necrosis Factor. Arthritis Rheum Vol. 56, No. 9, September 2007, pp 3033-3043 PMID: 17729298

19. Edd SN, Giori NJ, Andriacchi TP. The role of inflammation in the initiation of osteoarthritis after meniscal damage. J Biomech. 2015 Jun 1; 48(8):1420-6 doi: 10.1016/j.jbiomech.2015.02.035 PMID: 25798759

20. Rai MF, Patra D, Sandell LJ, Brophy RH. Relationship of Gene Expression in the Injured Human Meniscus to Body Mass Index: a Biological Connection between Obesity and Osteoarthritis. Arthritis Rheum. 2014 August; 66(8): 2152-2164.

21. Rai MF, Patra D, Sandell LJ, Brophy RH. Transcriptome Analysis of Human Injured Meniscus Reveals a Distinct Phenotype of Meniscus Degeneration with Aging. Arthritis Rheum. 2013 August; 65(8) 1-21

22. Petty CA, Lubowitz JH. Does arthroscopic partial meniscectomy result in knee osteoarthritis? A systematic review with a minimum of 8 years' follow-up. Arthroscopy. 2011 Mar; 27(3):419-24. doi: 10.1016/j. arthro.2010.08.016 PMID: 21126847

23. Cohen SB, Short CP, O'Hagan T, Wu HT, Morrison WB, Zoga AC. The effect of meniscal tears on cartilage loss of the knee: findings on serial MRIs. Phys Sportsmed. 2012 Sep; 40(3):66-76. doi: 10.3810/ psm.2012.09.1983 PMID: 23528623

24. Vundelinckx B, Vanlauwe J, Bellemans J. Long-term Subjective, Clinical, and Radiographic Outcome Evaluation of Meniscal Allograft Transplantation in the Knee. Am J Sports Med. 2014 Jul; 42(7):15929. doi: 10.1177/0363546514530092 PMID: 24758783

25. Rosso F, Bisicchia S, Bonasia DE, Amendola A. Meniscal allograft transplantation: a systematic review. Am J Sports Med. 2015 Apr; 43(4):998-1007. doi: 10.1177/0363546514536021 PMID: 24928760 
26. Smith NA, Parkinson B, Hutchinson CE, Costa ML, Spalding T. Is meniscal allograft transplantation chondroprotective? A systematic review of radiological outcomes. Knee Surg Sports Traumatol Arthrosc. 2015 Mar 19. [Epub ahead of print]

27. Messner $\mathrm{K}, \mathrm{Gao} J$. The menisci of the knee joint. Anatomical and functional characteristics, and a rationale for clinical treatment. J Anat. 1998 Aug; 193 (Pt 2):161-78. PMID: 9827632

28. Khetia EA, McKeon BP. Meniscal allografts: biomechanics and techniques. Sports Med Arthrosc. 2007 Sep; 15(3):114-20 PMID: 17700370

29. Verdonk R. Meniscal transplantation. Acta Orthop Belg. 2002 Apr; 68(2):118-27 PMID: 12050996

30. Moens K, Dhollander A, Moens P, Verdonk K, Verdonk R, Almqvist KF, et al. Meniscal transplantation: still experimental surgery? A review. Acta Orthop Belg. 2014 Sep; 80(3):403-13. PMID: 26280615

31. Reed ME, Villacis DC, Hatch GF III, Burke WS, Colletti PM, Narvy SJ, et al. 3.0-Tesla MRI and arthroscopy for assessment of knee articular cartilage lesions. Orthopedics. 2013 Aug; 36(8):e1060-4. doi: 10.3928/01477447-20130724-24 PMID: 23937754

32. Debeer $P$, Decorte R, Delvaux $S$, Bellemans J. DNA analysis of a transplanted cryopreserved meniscal allograft. Arthroscopy. 2000 Jan-Feb; 16(1):71-5. PMID: 10627349

33. Kellgren JH, Lawrence JS. Radiological assessment of osteo-arthrosis. Annals of the rheumatic diseases (1957) 16 (4): 494-502. PMID: 13498604

34. Elattar M, Dhollander A, Verdonk R, Almqvist KF, Verdonk P. Twenty-six years of meniscal allograft transplantation: is it still experimental? A meta-analysis of 44 trials. Knee Surg Sports Traumatol Arthrosc. 2011 Feb; 19(2):147-57. doi: 10.1007/s00167-010-1351-6 PMID: 21161170

35. Suárez Rancel, M, González Sierra MA. "Regression Diagnostic Using Local Influence: A Review," Communication in Statistics, Part A-Theory and Methods, 2001; 30, 799-813.

36. Pengas IP, Assiotis A, Nash W, Hatcher J, Banks J, McNicholas MJ. Total meniscectomy in adolescents: a 40-year follow-up. J Bone Joint Surg Br. 2012 Dec; 94(12):1649-54. doi: 10.1302/0301-620X. 94B12.30562 PMID: 23188906

37. Englund M, Roos EM, Lohmander LS. Impact of Type of Meniscal Tear on Radiographic and Symptomatic Knee Osteoarthritis. A Sixteen-Year Followup of Meniscectomy with Matched Controls. Arthritis Rheum Vol. 48, No. 8, August 2003, 2178-2187. PMID: 12905471

38. Khan M, Evaniew N, Bedi A, Ayeni OR, Bhandari M. Arthroscopic surgery for degenerative tears of the meniscus: a systematic review and meta-analysis. CMAJ. 2014 Oct 7; 186(14):1057-64. doi: 10.1503/ cmaj.140433 PMID: 25157057

39. Herrlin S, Hållander M, Wange P, Weidenhielm L, Werner S. Arthroscopic or conservative treatment of degenerative medial meniscal tears: a prospective randomised trial. Knee Surg Sports Traumatol Arthrosc. 2007 Apr; 15(4):393-401. PMID: 17216272

40. Katz JN, Losina E. Surgery versus physical therapy for meniscal tear and osteoarthritis. N Engl J Med. 2013 Aug 15; 369(7):677-8.

41. Roemer FW, Jarraya M, Niu J, Duryea J, Lynch JA, Guermazi A. Knee joint subchondral bone structure alterations in active athletes: a cross-sectional case-control study. Osteoarthritis Cartilage. 2015 Dec; 23(12):2184-90. doi: 10.1016/j.joca.2015.07.002 PMID: 26187571

42. Zaffagnini S, Marcheggiani Muccioli GM, Lopomo N, Bruni D, Giordano G, Ravazzolo G, et al. Prospective Long-Term Outcomes of the Medial Collagen Meniscus Implant Versus Partial Medial Meniscectomy: A Minimum 10-year Follow-Up Study. Am J Sports Med 2011 39: 977-85 doi: 10.1177/ 0363546510391179 PMID: 21297005

43. Sihvonen R, Paavola M, Malmivaara A, Ital A, Joukainen A, Nurmi H, et al. Arthroscopic Partial Meniscectomy versus Sham Surgery for a Degenerative Meniscal Tear. N Engl J Med 369;26 2515-24. doi: 10.1056/NEJMoa1305189 PMID: 24369076

44. Lyons MC, MacDonald SJ, Somerville LE, Naudie DD, McCalden RW. Unicompartmental versus total knee arthroplasty database analysis: is there a winner? Clin Orthop Relat Res. 2012 Jan; 470(1):8490. doi: 10.1007/s11999-011-2144-z PMID: 22038173

45. Bozic KJ, Durbhakula S, Berry DJ, Naessens JM, Rappaport K, Cisternas M, et al. Differences in patient and procedure characteristics and hospital resource use in primary and revision total joint arthroplasty: a multicenter study. J Arthroplasty. 2005 Oct; 20(7 Suppl 3):17-25 PMID: 16213998

46. Monllau JC, Alentorn-Geli E, Pelfort X, Torres R, Leal-Blanquet J, Hinarejos P. Meniscal Allograft Transplantation: Where are we Standing? J Transplant Technol Res 2014, 4:1 http://dx.doi.org/10. 4172/2161-0991.1000127

47. Samitier G, Alentorn-Geli E, Taylor DC, Rill B, Lock T, Moutzouros V, et al. Meniscal allograft transplantation. Part 2: systematic review of transplant timing, outcomes, return to competition, associated procedures, and prevention of osteoarthritis. Knee Surg Sports Traumatol Arthrosc. 2015 Jan; 23(1):32333. doi: 10.1007/s00167-014-3344-3 PMID: 25266230 